\title{
Streamers: Chemotactic Mutants of Dictyostelium discoideum with Altered Cyclic GMP Metabolism
}

\author{
By FIONA M. ROSS AND PETER C. NEWELL* \\ Department of Biochemistry, University of Oxford, South Parks Road, \\ Oxford OXI $3 Q U, U . K$.
}

(Received 8 May 1981)

\begin{abstract}
Mutants of Dictyostelium discoideum that developed huge aggregation streams in expanding clones were investigated using optical and biochemical techniques. Representatives of the six complementation groups previously identified $(\operatorname{stm} A-\operatorname{stm} F)$ were found to be similar to the parental wild-type strain XP55 in both the extent and timing of their ability to initiate and relay chemotactic signals and in the formation of cyclic AMP receptors and phosphodiesterases. The mutants differed from the wild-type in producing an abnormal chemotactic (movement) response visible using both dark-field optics with synchronously aggregating amoebae on solid substrata and light scattering techniques with oxygenated cell suspensions. Mutants of complementation group $\operatorname{stm} F$ showed chemotactic movement responses lasting up to $520 \mathrm{~s}$, rather than $100 \mathrm{~s}$ as seen in the parental and other strains. Measurements of cyclic GMP formed intracellularly in response to chemotactic pulses of cyclic AMP in $\operatorname{stm} F$ mutants showed that abnormally high concentrations of this nucleotide were formed within $10 \mathrm{~s}$ and were not rapidly degraded. A causal correlation between defective cyclic GMP metabolism and the altered chemotactic response is suggested, and a model is proposed that accounts for the formation of huge aggregation streams in clones of these mutants.
\end{abstract}

\section{INTRODUCTION}

Chemotaxis is an important feature of all living organisms, from bacteria searching for food to the complex movements of cells in the mammalian embryo. Considerable progress has been made in understanding the mechanism and control of chemotaxis in prokaryotes (see Hazelbauer, 1980, for a review), but our understanding of the workings of eukaryotic chemosensory systems is much more limited.

The unicellular eukaryote Dictyostelium discoideum has been much studied for its remarkable developmental cycle. Upon starvation the amoebae aggregate into clusters of about $10^{5}$ cells which then differentiate into stalk and spore cells and form a fruiting body. Chemotaxis is clearly an essential part of this organism's life cycle. In the vegetative phase folic acid is a chemoattractant which helps the amoebae to find their bacterial food (Pan et al., 1975), and negative chemotaxis induced by a small dialysable molecule of unknown structure helps population dispersal (Keating \& Bonner, 1977; Kakebeeke et al., 1979). In the developmental phase the amoebae move towards aggregation centres which emit regular pulses of cyclic AMP, and most of the studies on chemotaxis with this organism have been involved with this cyclic AMP-induced chemotactic system.

The pulsatile nature of the cyclic AMP signalling during aggregation may be visualized using sensitive dark-field optics. A field of aggregating amoebae on agar, viewed under dark-field, shows patterns of light and dark bands in concentric rings and spirals. Alcântara \& Monk (1974) have shown that the light bands are made up of elongated moving amoebae 
while the amoebae in the dark band are relatively rounded and stationary. By analysing time-lapse films of such banding patterns they also showed that amoebae move towards the cyclic AMP signal for about $100 \mathrm{~s}$ after each pulse. Using a microcapillary to deliver pulses of cyclic AMP to a field of starving amoebae of wild-type strain NC4, Robertson et al. (1972) showed that the cells became capable of responding chemotactically to a cyclic AMP signal at about $3 \mathrm{~h}$ after the removal of the food source, although they were not able to relay the signal until about $6 \mathrm{~h}$ (Gingle \& Robertson, 1976) and did not produce cyclic AMP autonomously until $10 \mathrm{~h}$ under the conditions used.

Specific receptors for cyclic AMP appear on the amoebal surface during development (Malchow \& Gerisch, 1974; Green \& Newell, 1975; Henderson, 1975). Scatchard plots of the binding of cyclic AMP at equilibrium indicate that there are both high (10 nM) and low $(150 \mathrm{nM})$ affinity forms of the receptors. Evidence at present favours a single type of receptor with variable affinity (Mullens \& Newell, 1978) although it is possible that there are two separate types of receptor molecule. The cell surface also bears phosphodiesterase enzyme molecules that, together with an excreted soluble form of the enzyme, destroy the cyclic AMP signal within a few seconds of its formation before it is able to saturate and block the cyclic AMP receptors (Chang, 1968; Pannbacker \& Bravard, 1972).

The membrane transduction system that links the cyclic AMP receptor to the intracellular system that brings about orientated movement is little understood. There is, however, good evidence that cyclic GMP is involved. Intracellular concentrations of this nucleotide rise to a peak within $10 \mathrm{~s}$ of stimulation with cyclic AMP in aggregating $D$. discoideum amoebae (Mato et al., 1977; Wurster et al., 1977). Folic acid produces a similar response in vegetative cells, and other species of slime mould react similarly to their specific chemoattractants (Mato \& Konijn, 1977; Wurster et al., 1978). Dicou \& Brachet (1980) have shown that there is an intracellular phosphodiesterase that is specific for cyclic GMP which is distinct from the phosphodiesterase that acts on both cyclic AMP and cyclic GMP. There are also specific cyclic GMP-binding proteins in the cell (Rahmsdorf \& Gerisch, 1978; Mato et al., 1979) although no functions (other than binding cyclic GMP) have as yet been ascribed to them. Quite recently, Mato \& Marin-Cao (1979) have demonstrated that there is a rapid transient methylation of a membrane protein in response to cyclic AMP stimulation. It is thus possible that cyclic GMP and methylation of membrane proteins are common factors in bacterial and slime mould chemotaxis.

This paper describes work on a class of 'aggregation pattern' mutants that are able to aggregate and develop but which form huge aggregation streams in expanding clones growing on lawns of bacteria. Twenty-four such 'streamer' mutants have been analysed genetically (Ross \& Newell, 1979) and have been assigned to six complementation groups (stmA to $s t m F$ ). (The original data, which placed four of these mutants in a seventh complementation group, $\operatorname{stm} G$, has recently been found to be in error and following further complementation studies these four mutants have been reallocated to $\operatorname{stm} F$ which now contains six members.) Because the streamer mutants formed very large territories, collecting amoebae from over a wide area, it was originally considered likely that their primary defect lay in the initiation of aggregation centres. The work described below indicates, however, that the defect present in these mutants is connected with their chemotactic ability and that this leads to a restriction of aggregation centre initiation under conditions of clonal growth.

\section{METHODS}

Media and chemicals. The composition and method of preparation of nutrient (SM) agar were as described previously (Sussman, 1966; Mosses et al., 1975). $\mathrm{KK}_{2}$ agar contained (per litre): $\mathrm{KH}_{2} \mathrm{PO}_{4}, 1.125 \mathrm{~g} ; \mathrm{K}_{2} \mathrm{HPO}_{4}$, $0.335 \mathrm{~g} ; \mathrm{MgCl}_{2} \cdot 6 \mathrm{H}_{2} \mathrm{O}, 0.5 \mathrm{~g}$. Normally this medium was autoclaved at $121^{\circ} \mathrm{C}$ for $15 \mathrm{~min}$, but when it was required for photographic experiments it was boiled rather than autoclaved as this gave a clearer gel.

Strains. All Dictyostelium discoideum stocks used are derivatives of NC4 (Raper, 1935). The isolation and characterization of the streamer mutants was described by Ross \& Newell (1979). For this study only mutants 
in an XP55 background were chosen. Strains NP294, NP371, NP383, NP370 and NP387 were used as representatives of complementation groups $s t m A$ to $s t m E$, respectively; mutant NP377 (bearing stmF411) was generally employed as representative of complementation group $\mathrm{stm} F$, but mutant NP368 (bearing $\mathrm{stmF406)}$ was employed in some experiments where indicated.

Maintenance of stocks. Standard procedures were employed for the handling of amoebae and the preservation of spores on silica gel (Williams et al., 1974). In addition, a secondary stock of each strain was kept as amoebae frozen in horse serum (containing $5 \%, \mathrm{v} / \mathrm{v}$ dimethyl sulphoxide) in plastic straws immersed in liquid nitrogen. A fresh straw was opened approximately every 2 months.

Cyclic AMP equilibrium binding assay. Cells were allowed to develop on $\mathrm{KK}_{2}$ plates held at $7^{\circ} \mathrm{C}$ overnight, then returned to $22^{\circ} \mathrm{C}$. The cells were harvested when streaming was well under way. The assay used was as described by Green \& Newell (1975) except that Nuclepore polycarbonate filters (Sterilin Ltd, Teddington, Middx) were used instead of Millipore membrane filters (Mullens \& Newell, 1978). The incubation time for each sample was 2 min.

Cyclic AMP phosphodiesterase assays. Cells were allowed to develop at $22^{\circ} \mathrm{C}$ on $\mathrm{KK}_{2}$ agar at $5 \times 10^{7}$ cells per plate. For cell-bound phosphodiesterase assay, the cells were harvested in 17 mM-Sorensen's phosphate buffer, washed and resuspended at $10^{7}$ cells $\mathrm{ml}^{-1}$. For extracellular phosphodiesterase assay, the entire contents of an agar plate (agar and amoebae) were centrifuged through Swiss Nylon bolting cloth (John Stanier Ltd, Manchester) (Gerisch, 1976) for $5 \mathrm{~min}$ at $150 \mathrm{~g}$ and the supernatant was retained for assay. The assay was the same for both types of phosphodiesterase. A $40 \mu \mathrm{l}$ sample was shaken for $15 \mathrm{~min}$ at $23^{\circ} \mathrm{C}$ with $10 \mu \mathrm{l}$ of a $250 \mu \mathrm{M}$-cyclic [ $\left.{ }^{3} \mathrm{H}\right] \mathrm{AMP}$ solution $\left[25-30 \mathrm{Ci} \mathrm{mmol}^{-1}\left(0.93-1.11 \mathrm{TBq} \mathrm{mmol}^{-1}\right)\right.$, obtained from Amersham, diluted $1: 200$ with unlabelled cyclic AMP]. The reaction was stopped by adding $10 \mu \mathrm{l}$ of $55 \%(\mathrm{w} / \mathrm{v})$ trichloroacetic acid containing cyclic AMP, AMP and adenosine (each at $15 \mathrm{mg} \mathrm{ml}^{-1}$ ). A $10 \mu \mathrm{l}$ sample from each assay tube was spotted on to Whatman no. 1 filter paper and the papers were developed in a descending chromatography tank for $12 \mathrm{~h}$ in $95 \%(\mathrm{v} / \mathrm{v})$ ethanol/1 M-ammonium acetate, $0.01 \mathrm{M}$-EDTA $(70: 30, \mathrm{v} / \mathrm{v})$ solvent. The spots were detected with short-wave u.v. light, cut out and counted in a toluene scintillant containing $4 \mathrm{~g} \mathrm{PPO} \mathrm{I}^{-1}$ and $50 \mathrm{mg}$ POPOP $1^{-1}$.

Light scattering. Cells were allowed to develop on plates as above. At the appropriate time they were harvested and resuspended at $3 \times 10^{8}$ cells $\mathrm{ml}^{-1}$ in $17 \mathrm{~mm}$-phosphate buffer, pH 6.2. Light scattering experiments were then carried out according to the method of Gerisch \& Hess (1974).

Cyclic AMP assays. Samples for assaying total cellular cyclic AMP were taken from the cuvette of light scattering experiments at $10 \mathrm{~s}$ intervals. All reactions were stopped by adding $4 \mathrm{M}$-perchloric acid. The $\mathrm{pH}$ was adjusted to 7.5 with $\mathrm{KOH}$, and the precipitate was sedimented by centrifuging for 1 min in a Beckman microcentrifuge. The supernatant was assayed using the Amersham cyclic AMP assay kit. An alternative method of stopping the reaction was to use ice-cold ethanol/ $\mathrm{HCl}(60: 1, \mathrm{v} / \mathrm{v})$ followed by ether extraction.

Cyclic GMP assays. Cells were allowed to develop in $17 \mathrm{~mm}$-phosphate buffer in shaken suspension at $23{ }^{\circ} \mathrm{C}$. At $8 \mathrm{~h}$ the cells were harvested and resuspended at $10^{8} \mathrm{ml}^{-1}$. Thereafter they were treated as described by Mato et al. (1977). Assays were carried out using the Amersham cyclic GMP assay kit. Both cyclic AMP and cyclic GMP assays were counted in toluene/Triton X-100/PPO scintillant.

Photography. A high-sensitivity, dark-field optical system was built based on the design of Trevan (Gross et al., 1976). An annulus of $2 \mathrm{~mm}$ width and $25 \mathrm{~mm}$ inner diameter was used with a green filter and diffusing screen. The biconvex illuminator lenses were $120 \mathrm{~mm}$ in diameter and of approximately $120 \mathrm{~mm}$ focal length. A Nikon FM single-lens reflex camera was positioned above the specimen holder in a moveable mount attached to light-excluding bellows. A Nikon MD-12 motor drive and Intervalometer were used for time-lapse studies.

\section{RESULTS}

To avoid complications due to differences in genetic background, the studies described below used representative mutants from each of the six streamer complementation groups, $\operatorname{stm} A$ to $\operatorname{stm} F$, which had been selected in the same parental strain, XP55. [For simplicity the streamer phenotype codes (StmA to StmF) are used instead of mutant numbers; the relevant mutant numbers are given in Methods.]

Mutant phenotype in clones and synchronously developing populations. When growing as clones on lawns of bacteria, the streamer mutants are characterized by forming huge streams during aggregation, whereas the parental strain XP55 and other strains form scarcely discernible streams under these high population density conditions. In contrast to this behaviour, when mutant amoebae grown on bacterial lawns were harvested, washed free of bacteria and 
replated on to non-nutrient $\mathrm{KK}_{2}$ plates, they aggregated with similar timing to the parental strain and did not form abnormally large territories.

The significant difference in conditions between clones and non-nutrient plates would seem to be the synchronous stimulation of development on non-nutrient plates compared to the asynchrony seen in growing clones. As a clone grows outwards there is a ring of vegetative amoebae at the periphery, and starving and progressively more developed cells towards the centre of the clone. Such asynchrony may be mimicked under particular conditions with amoebae starving on $\mathrm{KK}_{2}$ agar plates. To attain good synchrony of development the agar plates were normally carefully poured on a flat surface and the plates were air-dried after plating the starving amoebae. If the agar was poured on a very slightly tilted surface and the subsequent drying-in was incomplete, the amoebae in the lower (and slightly damper) part of the agar developed slightly more slowly than those in the higher part. It is noteworthy that under these conditions the mutants, but not to any great extent the controls, formed large streams that extended from the high to the low part of the agar. We deduce that the asynchrony, rather than any special effects of growth on bacterially inoculated plates, is responsible for the streaming phenotype.

Centre transplantation tests. A possible explanation of the difference in phenotype between asynchronous and synchronous conditions might have been that the mutants required significantly different intervals between becoming able to relay and gaining the ability to signal spontaneously. This possibility was ruled out by experiments involving transplantation of mutant and parental centres. When centres of different ages from mutant and parental aggregates were carefully interchanged (with a microcapillary) during the period 3 to $8 \mathrm{~h}$ after the onset of starvation, no differences in the time required for the starving mutants to become chemotactically competent or able to relay or initiate signals could be detected. (These properties were simply determined by screening visually for local or field-wide chemotaxis.) It was also noted that in strain XP55 and all the streamer mutants the ability to relay signals developed within $1 \mathrm{~h}$ of the ability to initiate signal pulses autonomously. This finding differs from the reports of Gingle \& Robertson (1976) in which the ability to initiate autonomous signals developed about $4 \mathrm{~h}$ after the ability to relay.

Response to cyclic AMP in oxygenated suspensions. In order to observe the effects of cyclic AMP pulses on the mutants in a quantitative way, the changes in light scattering seen in dense suspensions of starving amoebae were monitored spectrophotometrically at $405 \mathrm{~nm}$ using the method of Gerisch \& Hess (1974). In the early stages of development, XP55 and other (wild-type) strains responded to a $100 \mathrm{nM}$ pulse of cyclic AMP with a monophasic decrease in light scattering which became biphasic as aggregation proceeded. The mutants also gave a monophasic response at early times, but although the response did change with developmental time they never produced a normal second peak (Fig. 1). The start of the second response was delayed in all cases by 30 to $60 \mathrm{~s}$ and it levelled off at the minimum without returning to the higher basal level of the wild-type. In most cases the amplitude of the first peak was greater in the mutants than in XP55. A second $100 \mathrm{nM}$ pulse of cyclic AMP given less than 1 min after the first one produced another similar first wave response in the mutants despite the failure of the baseline to return to normal. The results suggested that some aspect of the signalling system and more particularly the cells' response to signals was altered in the mutants. The components of the signalling system were therefore investigated in more detail.

Cyclic AMP receptors. Measurements were made of the binding of cyclic [ $\left.{ }^{3} \mathrm{H}\right] \mathrm{AMP}$ to whole starving amoebae and the equilibrium binding characteristics of XP55 and mutants were determined using Scatchard plot analysis. The plots for XP55, StmD and StmF are shown in Fig. 2. For mutants StmA, B, C and E the lines were of almost identical slope and axis intercepts to those shown for StmD. These plots show that strain XP55 has fewer highaffinity binding sites than other strains derived from NC4 (Green \& Newell, 1975; Mullens \& Newell, 1978) but that their affinity is considerably higher (in the range 0.1 to $0.5 \mathrm{~nm}$ 


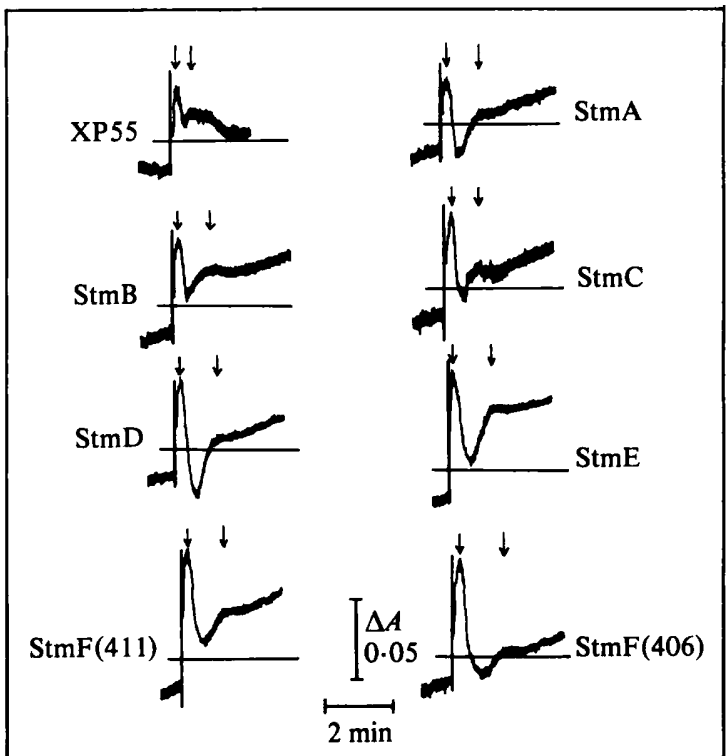

Fig. 1. Responses of oxygenated suspensions of amoebae of strain XP55 and the streamer mutants to pulses of cyclic AMP. Pulses of $100 \mathrm{nM}$-cyclic AMP (final concentration) were given after $8 \mathrm{~h}$ of starvation and the amoebal responses due to changes in light scattering properties were measured spectrophotometrically at $405 \mathrm{~nm}$. The time of addition of the cyclic AMP pulse is shown by the vertical line in each trace and the timing of the first and second response peaks by arrows. Due to dilution of the cell suspension by the added pulses of cyclic AMP the baseline of the traces is shifted upwards to the position shown by the horizontal line.

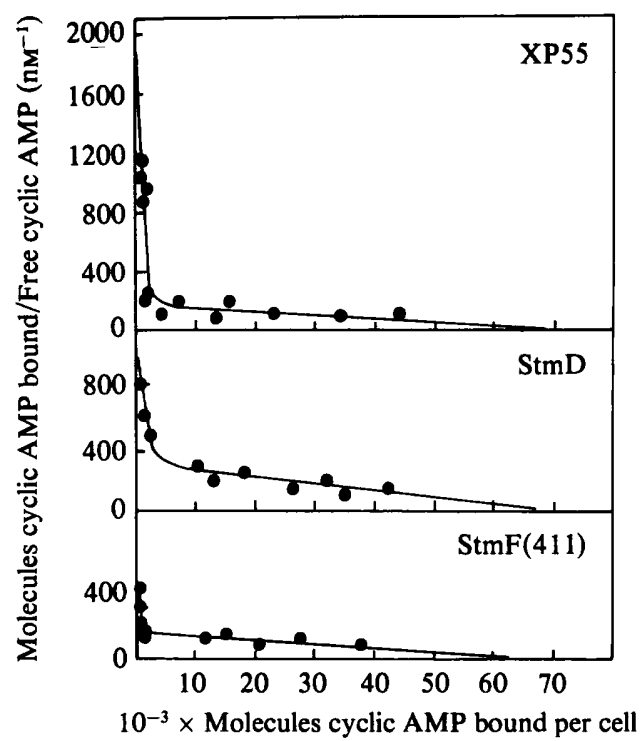

Fig. 2. Scatchard plots of cyclic $\left[{ }^{3} \mathrm{H}\right] \mathrm{AMP}$ binding at equilibrium to starving amoebae of strain XP55 and streamer mutants StmD and $\operatorname{StmF}(411)$.

compared with $10 \mathrm{nM}$ ). The precise numbers of receptors showing this high affinity varied from one experiment to another, however, and sometimes they could be barely detected. It is not clear what conditions caused the differences. The low affinity sites also differed from 


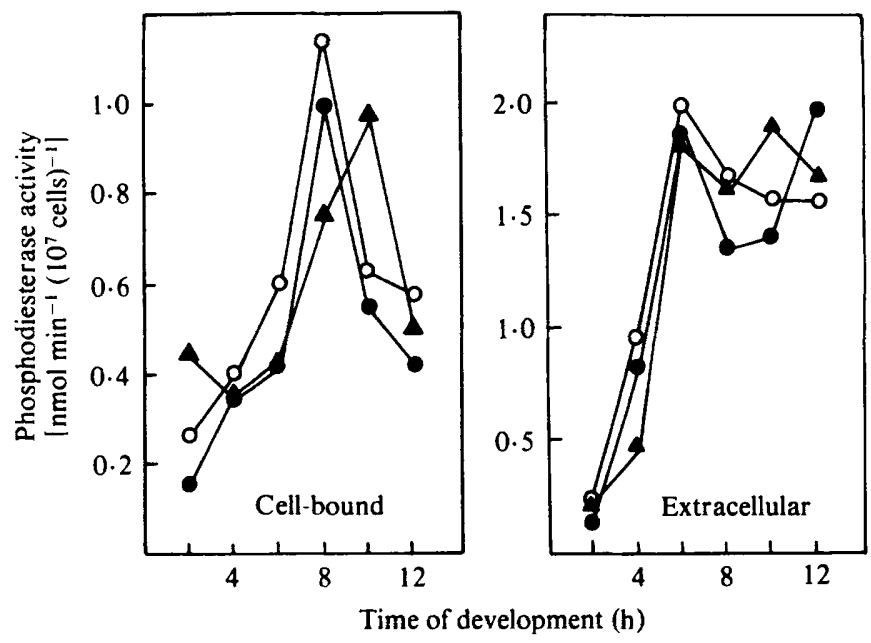

Fig. 3. Cell-bound and extracellular phosphodiesterase activities (measured at $23{ }^{\circ} \mathrm{C}$ ) of strain XP55

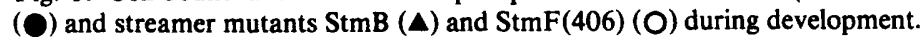

those in previously reported studies, there being about half the number with twice the $K_{\mathrm{m}}$ value compared with strains AX2 or X22. The cyclic AMP receptors in the mutants tended to have slightly higher $K_{\mathrm{m}}$ values for both affinities than their parent, but the differences were small and unlikely to account for their altered behaviour. It was particularly difficult to demonstrate the presence of high affinity sites in StmF mutants, but as very similar results were sometimes obtained for XP55 it is probable that this is not significant for the streaming phenotype. It is of interest that such low numbers of high affinity sites were present in strains which could clearly both move towards cyclic AMP and relay the signal.

Cyclic AMP phosphodiesterases. Assays for cell-bound and extracellular phosphodiesterases in strain XP55 revealed that both activities were slightly lower throughout development than in other NC4-derived strains (Fig. 3), but the pattern of development and the activities achieved in the mutants were essentially the same as in their parent, as can be seen for StmB and StmF in Fig. 3. The remaining mutants gave similar results to those shown. It seems very unlikely, therefore, that cyclic AMP phosphodiesterases play any part in the altered mutant phenotype.

Signal amplification. An attempt was made to demonstrate amplification of a cyclic AMP signal in oxygenated cell suspensions. Using the axenic strain AX2, Roos et al. (1975) and Gerisch \& Wick (1975) reported that an applied cyclic AMP pulse was amplified by starving amoebal suspensions and that the synthesis and release of cyclic AMP was in phase with the second light scattering response. However, we have been unable to show the amplification of such an applied pulse using this technique in various ways in any strain examined, including strain AX2, although the presence of the added cyclic AMP and its rapid degradation within a few seconds could be readily detected. It is not altogether clear why no amplification could be seen, although it is possible that the applied pulse was too brief to stimulate a measurable relay response. From the work of Devreotes \& Steck (1979) using a concentration clamp technique, a transitory pulse would be expected to give only a very brief period of stimulation of cyclic AMP synthesis, as these workers found that cyclic AMP synthesis lasted only as long as the incoming signal and that the stimulation had to be present continuously to sustain cyclic AMP secretion.

Initiation and relay on agar. One of the clearest ways of demonstrating and measuring the ability of amoebae to initiate and relay cyclic AMP signals is by observing lawns of starving amoebae on agar surfaces using dark-field optics. Incubation for the first $16 \mathrm{~h}$ of starvation at $7^{\circ} \mathrm{C}$ produces highly synchronized populations that, when shifted to $22^{\circ} \mathrm{C}$, 

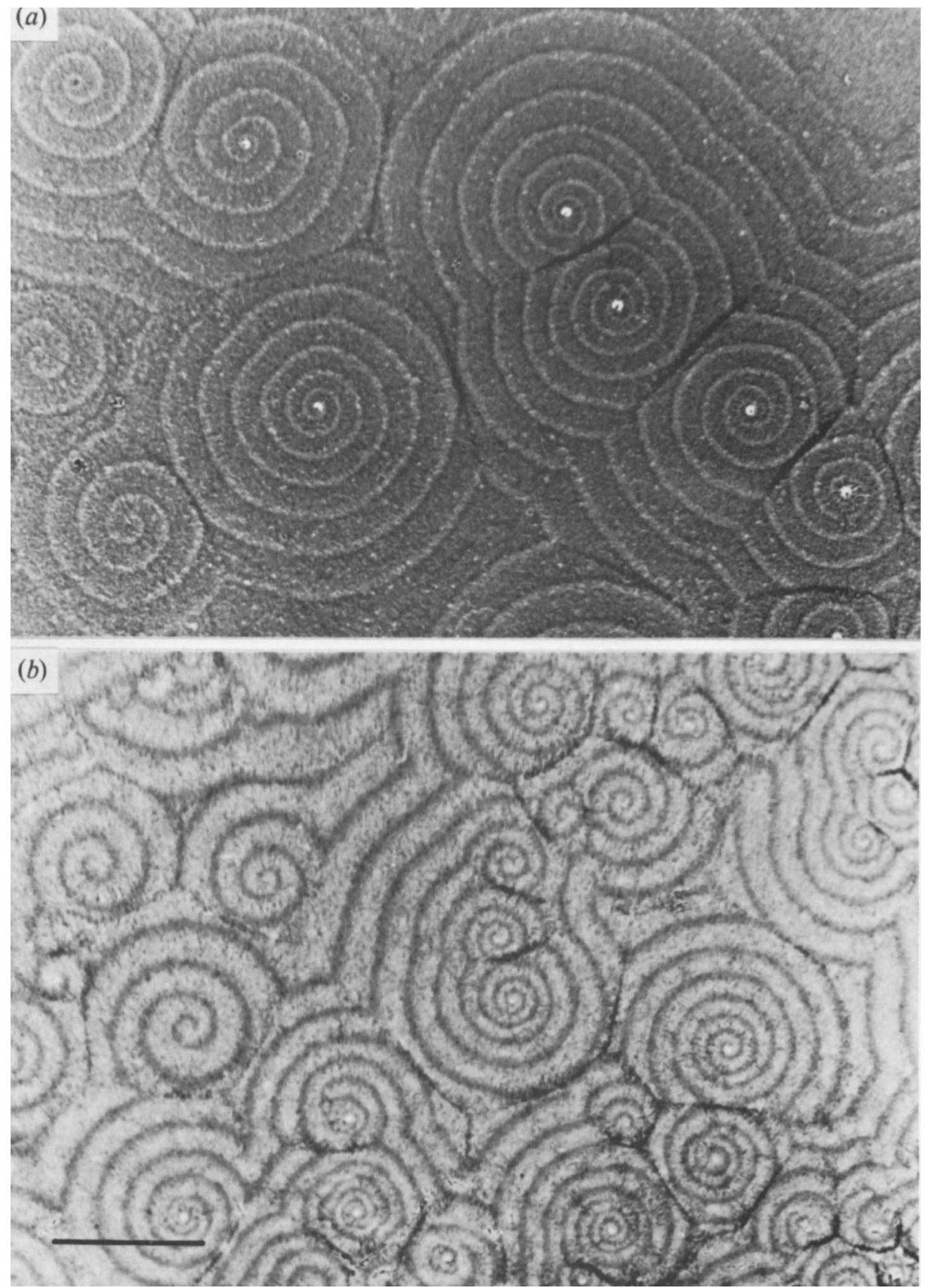

Fig. 4. Aggregating fields of parental strain XP55 (a) and streamer mutant StmF(406) (b) on agar observed with dark-field optics. The moving bands of amoebae appear bright and the stationary amoebae appear dark under these conditions. Note the wide movement bands with StmF compared with XP55. The bar marker represents $5 \mathrm{~mm}$.

show clear light and dark bands. Time-lapse $35 \mathrm{~mm}$ photography allowed subsequent analysis of the wave velocity as well as the band widths and signal periodicities. Figures 4 and 5 show photographs of XP55 and StmF amoebae on $\mathrm{KK}_{2}$ agar. The banding pattern of 

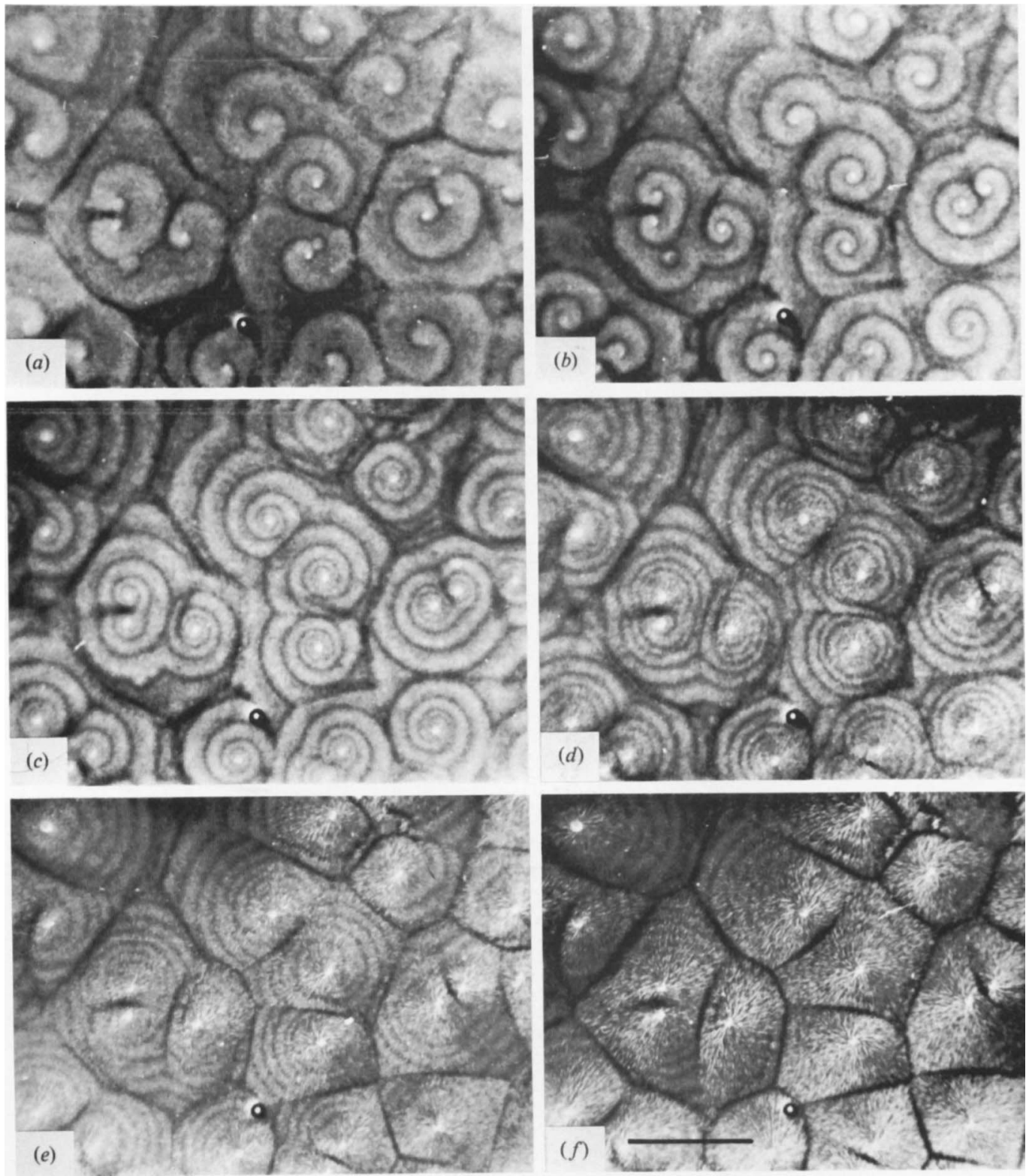

Fig. 5. Time-lapse photographs of a field of starving amoebae of $\operatorname{StmF}(411)$ seen with dark-field optics. The frames (taken at approximately 10 min intervals) show mainly spiral waves of steadily increasing frequency that lead to the formation of defined aggregation territories and finally to streams of amoebae heading towards the signalling centres. The small circle in the lower middle of each frame is a marker bubble in the agar. The bar marker represents $10 \mathrm{~mm}$.

XP55 was extremely similar to that of NC4 (Alcântara \& Monk, 1974) with narrow light bands moving out from the centres either as spirals or concentric rings. With good resolution and highly synchronous populations of amoebae a very narrow, very dark band could be seen immediately preceding each light band. The periodicity of the signal, the wave velocity and the duration of the movement period (calculated from the width of the bright bands) were similar to those of NC4. Of particular importance for the following discussion is the duration 


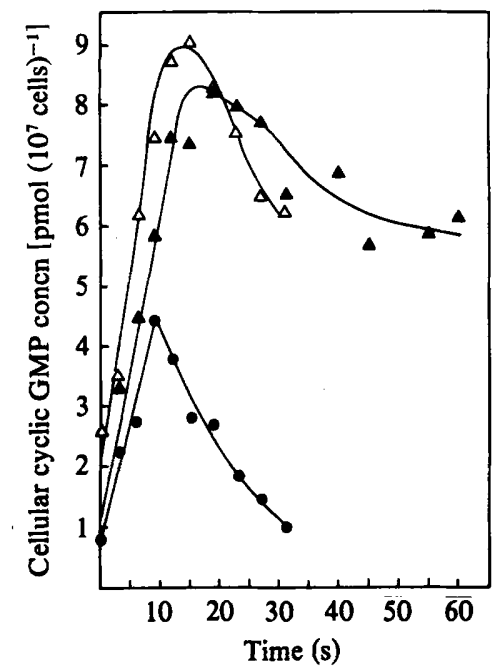

Fig. 6. Changes in the cellular concentration of cyclic GMP in response to a $50 \mathrm{nM}$ pulse of cyclic AMP given at $t_{0}$ to amoebae of strain XP55 (O), and streamer mutants $\operatorname{StmF}(411)(\Delta)$ and $\operatorname{StmF}(406)(\triangle)$ after $8 \mathrm{~h}$ of starvation. The data represent the average of four experiments.

of the movement period which was found for strain XP55, as for other wild-type strains, to be $100 \mathrm{~s}$. The photographs of the StmF mutant almost appear to be negatives of the parental strain; there are narrow dark bands separated by broad light bands. Time-lapse photographs of a field of StmF amoebae are shown in Fig. 5. Measurements from these photographs showed that periodicities and wave velocities were normal but that the movement step was prolonged. The length of time for which these amoebae moved in response to a cyclic AMP signal was related to the periodicity of the signal and could be as long as $520 \mathrm{~s}$ when there was a long interval between pulses. Because all measurements other than those directly involved with the chemotactic response were normal in these strains it appears that they are perfectly capable of receiving and relaying signals in the normal way and it is only the chemotactic response which is altered.

Regular banding patterns were not easily seen in mutants belonging to the other streamer complementation groups, although some bands could be seen if the cells were incubated at $7^{\circ} \mathrm{C}$ for $20 \mathrm{~h}$ or more. Even then, successive waves from a single centre were rarely seen sufficiently clearly to make reliable measurements. However, the light bands produced under these conditions were usually significantly broader than those of XP55 and narrow dark bands were present though often indistinct. Thus the chemotactic response that is characteristic of StmF seems to be present in these mutants but is easily masked by their inherent asynchrony.

Cyclic GMP metabolism. As studies of Wurster et al. (1977) and Mato et al. (1977) have implicated cyclic GMP in the chemotactic response, the streamer mutants were tested for the production of this nucleotide in response to a $50 \mathrm{nM}$ pulse of cyclic AMP. The results obtained for strain XP55 and two different StmF mutants are shown in Fig. 6. The other mutants gave results similar to those of XP55. The peak level determined for XP55 is about half that for NC4 as reported by Mato et al. (1977), but this difference is probably due to differences of assay technique as strain HL100 (obtained from Dr W. F. Loomis), which is a strain independently derived from NC4 without mutagenesis, showed similar peak levels to XP55 in our experiments. The interesting outcome from these studies is that the cyclic GMP formed after cyclic AMP stimulation in StmF mutants was found to be substantially higher than in XP55 and was clearly not degraded rapidly but persisted for at least a minute at elevated levels. 


\section{DISCUSSION}

The results presented here indicate that streamer mutants are altered in their chemotactic response to a cyclic AMP signal. No evidence could be found for any abnormality in cyclic AMP binding, phosphodiesterase activity or the developmental timing of any of the aggregative competences.

The normal first movement response of a chemotactically competent amoeba is to round up (Wurster et al., 1978). This rounding up (aptly termed 'cringing' by Futrelle et al., 1980), which may be seen as a narrow dark band adjacent to the light band when amoebae develop synchronously on agar, is exaggerated or prolonged in the StmF streamer mutants. The width of this narrow dark band correlates well with the duration of the first light scattering peak in both the parental and mutant strains, and we believe that this correlation is indicative of the two phenomena being manifestations of the same response. Unlike normal strains, once the streamer mutants have started moving they do not stop after $100 \mathrm{~s}$ but may continue for as long as $520 \mathrm{~s}$ or until another cyclic AMP signal reaches them. This results in the broad light band on agar and may also be visualized as the incomplete second response in light scattering measurements. From both the photographic evidence and the light scattering measurements employing double pulses it appears that these mutant amoebae respond normally to a second cyclic AMP pulse even though they are still moving in response to the first one. They round up immediately on receipt of the second pulse and show their characteristic light scattering profile. From dark-field studies the mutants are obviously fully capable of relaying the signal outwards. Clearly, being currently in the state of responding to one chemotactic stimulus is no bar on the amoebae chemotactically responding to, and relaying, another.

In order to explain the streaming phenotype in terms of the observed chemotactic aberration we wish to put forward the following model. We postulate that a chemotactically moving amoeba, although apparently capable of relaying a signal, cannot become an autonomous signaller. In view of the finding of Shaffer (1961) that 'Founder' cells of Polysphondylium violaceum stop and round up before apparently initiating centres, this postulate does not seem unreasonable. If this is the case, then when streamer mutants are grown as clones on agar in association with bacteria or on non-synchronized (tilted) $\mathrm{KK}_{2}$ plates, the first centres to be formed would attract nearby amoebae chemotactically before the amoebae became competent to initiate centres themselves. Because the mutant amoebae would be induced to move towards the centres for a far higher proportion of the time than their wildtype counterparts, it would be much less probable that any of them could become new autonomously signalling centres. This would result in long streams being formed before a new centre was eventually established. In contrast, in synchronously starving cultures all centres would be formed simultaneously (in both wild-type and mutants) and there would be no possibility of seeing the increased gathering power of mutant centres, although the mutant banding pattern would still be present.

The most exciting finding from these studies is the discovery that the StmF mutants show aberrant cyclic AMP-induced cyclic GMP accumulation. This is further evidence for the involvement of cyclic GMP in the chemotactic response, and confirms that the mutant defect is in the response to the signal rather than that the signal itself is altered. Further study of these mutants could throw light on such questions as whether there is a single cyclic GMP stimulatory mechanism which is triggered by both cyclic AMP and folic acid at different stages of development, whether cyclic GMP is involved in the adaptation of the cells to the signal and whether the altered control of cyclic GMP in these mutants is the result of increased guanylate cyclase activation or decreased cyclic GMP phosphodiesterase activity.

Although the other streamer mutants show normal cyclic AMP-induced cyclic GMP accumulation, it is possible that some other aspect of this system is affected (such as the affinity of the internal cyclic GMP-binding proteins) that would give a similar phenotype to 
a mutant with elevated cyclic GMP accumulation. It seems possible that the streamer mutants could be an important tool in unravelling the problem of chemotaxis in the cellular slime moulds.

We wish to thank Barrie Coukell, Isabel Mullens, David Ratner and Jeannie Wailace for helpful discussions, and Steven McClue and Jane Hardwicke for excellent technical assistance. Part of this work was supported by an MRC studentship to F.M.R.

\section{REFERENCES}

AlCÂNTARA, F. \& Monk, M. (1974). Signal propagation during aggregation in the slime mould Dictyostelium discoideum. Journal of General Microbiology 85, 321-334.

Chang, Y. Y. (1968). Cyclic 3',5'-adenosine monophosphate phosphodiesterase produced by the slime mold Dictyostelium discoideum. Science 160, $57-59$.

Devreotes, P. N. \& Steck, T. L. (1979). Cyclic 3',5'-AMP relay in Dictyostelium discoideum. II. Requirements for the initiation and termination of the response. Journal of Cell Biology 80, 300-309.

Dicou, E. L. \& BRAChET, P. (1980). A separate phosphodiesterase for the hydrolysis of cGMP in growing Dictyostelium discoideum amoebae. European Journal of Biochemistry 109, 507-514.

Futrelle, R. P., McKee, W. G. \& Traut, J. (1980). Response of Dictyostelium discoideum to localised cAMP stimuli: computer analysis of cell motion. Journal of Cell Biology 87, 57a.

Gerisch, G. (1976). Extracellular cAMP phosphodiesterase regulation in agar plate cultures of Dictyostelium discoideum. Cell Differentiation 5, 21-25.

Gerisch, G. \& Hess, B. (1974). Cyclic AMP controlled oscillations in suspended Dictyostelium cells: their relation to morphogenetic cell interactions. Proceedings of the National Academy of Sciences of the United States of America 71, 2118-2122.

GeRISCH, G. \& WICK, U. (1975). Intracellular oscillations and release of cyclic AMP from Dictyostelium cells. Biochemical and Biophysical Research Communications 65, 364-370.

Gingle, A. R. \& Robertson, A. (1976). The development of the relaying competence in Dictyostelium discoideum. Journal of Cell Science 20, 21-27.

Green, A. A. \& Newell, P. C. (1975). Evidence for the existence of two types of cAMP binding sites in aggregating cells of Dictyostelium discoideum. Cell 6, 129-136.

Gross, J. D., Peacey, M. J. \& Trevan, D. J. (1976). Signal emission and signal propagation during early aggregation in Dictyostelium discoideum. Journal of Cell Science 22, 645-656.

HAzelbauer, G. L. (1980). Bacterial chemotaxis: molecular biology of a sensory system. Endeavour 4, 67-73.

Henderson, E. J. (1975). The cyclic adenosine3',5'-monophosphate receptor of Dictyostelium discoideum. Journal of Biological Chemistry 250, 4730-4736.

Kakebeeke, P. J., De WIt, R. J. W., KohtZ, S. D. \& KoniJn, T. M. (1979). Negative chemotaxis in
Dictyostelium and Polysphondylium. Experimental Cell Research 124, 429-434.

KeATING, M. T. \& BonNER, J. T. (1977). Negative chemotaxis in cellular slime molds. Journal of Bacteriology 130, 144-147.

Malchow, D. \& Gerisch, G. (1974). Short-term binding and hydrolysis of cyclic $3^{\prime}, 5^{\prime}$-adenosine monophosphate by aggregating Dictyostelium cells. Proceedings of the National Academy of Sciences of the United States of America 71, 24232427.

Mato, J. M. \& Konun, T. M. (1977). Chemotactic signal and cGMP accumulation in Dictyostelium. In Development and Differentiation in Cellular Slime Moulds, pp. 93-104. Edited by P. Cappuccinelli \& J. Ashworth. Amsterdam: Elsevier/North Holland.

Mato, J. M. \& MARIN-CAO, D. (1979). Protein and phospholipid methylation during chemotaxis in Dictyostelium discoideum and its relationship to calcium movements. Proceedings of the National Academy of Sciences of the United States of America 76, 6106-6109.

Mato, J. M., Krens, F. A., Van HaAstert, P. J. M. \& KoniJn, T. M. (1977). 3',5'-cyclic AMP-dependent $3^{\prime}, 5^{\prime}$-cyclic GMP accumulation in Dictyostelium discoideum. Proceedings of the National Academy of Sciences of the United States of America 74, 2348-2351.

Mato, J. M., Woelders, H. \& Konin, T. M. (1979). Intracellular cyclic GMP-binding proteins in cellular slime molds. Journal of Bacteriology 137, 169-172.

Mosses, D., Williams, K. L. \& Newell, P. C. (1975). The use of mitotic crossing over for genetic analysis in Dictyostelium discoideum: mapping of linkage group II. Journal of General Microbiology 90, 247-259.

Mullens, I. A. \& Newell, P. C. (1978). cAMP binding to cell surface receptors of Dictyostelium. Differentiation 10, 171-176.

Pan, P., Hall, E. M. \& BonNer, J. T. (1975). Determination of the active portion of the folic acid molecule in cellular slime mold chemotaxis. Journal of Bacteriology 122, 185-191.

Pannbacker, R. G. \& Bravard, L. J. (1972). Phosphodiesterase in Dictyostelium discoideum and the chemotactic response to cyclic adenosine monophosphate. Science 175, 1014-1015.

RAHMSDORF, H. J. \& Gerisch, G. (1978). Specific binding proteins for cAMP and cGMP in Dictyostelium discoideum. Cell Differentiation 7, 249-257.

RAPER, K. B. (1935). Dictyostelium discoideum, a new species of slime mold from decaying forest 
leaves. Journal of Agricultural Research 50, Sussman, M. (1966). Biochemical and genetic methods 135-147.

Robertson, A., Drage, D. J. \& Cohen, M. H. (1972). Control of aggregation in Dictyostelium discoideum by an external periodic pulse of cyclic adenosine monophosphate. Science 175, 333-335.

Roos, W., Nanjundiah, V., Malchow, D. \& GERISCH, G. (1975). Amplification of cyclic-AMP signals in aggregating cells of Dictyostelium discoideum. FEBS Letters 53, 139-142.

Ross, F. M. \& NewELL, P. C. (1979). Genetics of aggregation pattern mutations in the cellular slime mould Dictyostelium discoideum. Journal of General Microbiology 115, 289-300.

SHAFFER, B. M. (1961). The cells founding aggregation centres in the slime mould Polysphondylium tiolaceum. Journal of Experimental Biology 38, 833-849. in the study of cellular slime mold development. Methods in Cell Physiology 2, 397-410.

Williams, K. L., Kessin, R. H. \& Newell, P. C. (1974). Parasexual genetics in Dictyostelium discoideum: mitotic analysis of acriflavin resistance and growth in axenic medium. Journal of General Microbiology 84, 59-69.

Wurster, B., Schubiger, K., Wick, U. \& Gerisch, G. (1977). Cyclic GMP in Dictyostelium discoideum: oscillations and pulses in response to folic acid and cAMP signals. FEBS Letters 76, 141-144.

Wurster, B., Bozzaro, S. \& Gerisch, G. (1978). cGMP regulation and responses of Polysphondylium violaceum to chemoattractants. Cell Biology International Reports 2, 61-69. 